\title{
XXVII Workshop on Nuclear Physics in Brazil
}

\section{Foreword}

The XXVII Workshop on Nuclear Physics in Brazil took place in Santos, SP, Brazil, from 7-11 September 2004, with 153 participants. The program included 12 plenary invited talks, 2 colloquia, 25 oral presentations (in parallel sessions), and 105 poster presentations. The contributions covered the subjects of Nuclear Reactions, Nuclear Structure, Nuclear Matter at High Energy Densities, Nuclear Astrophysics, Nuclei far from Stability, Particle-Nucleon Interactions, Photonuclear Reactions, Applied Nuclear Physics, and Nuclear Instrumentation, that were roughly divided into four categories, Nuclear Reactions, Nuclear Structure, Nucleon Structure and Interactions, and Applied Physics and Instrumentation. The authors of the contributions were invited to submit an original article for publication in this special issue of the Braz. J. Phys., which is entirely dedicated to the proceedings of the Workshop. We would like to thank Prof. Silvio R. A. Salinas, Editor, for opening this privileged space for our proceedings. We would also like to acknowledge the financial support of FAPESP, CNPq, CNEN, FAPERJ, FINEP, CAPES, CLAF, and FUNCITEC.

\author{
Hugo Schelin, \\ José Ricardo Marinelli, \\ Marcos N. Martins, and \\ Nelson Carlin \\ Guest Editors
}

